\title{
Optical coherence tomography for quantitative surface topography
}

\author{
Sergio Ortiz, ${ }^{1,2, \star}$ Damian Siedlecki, ${ }^{1,3}$ Laura Remon, ${ }^{1}$ and Susana Marcos ${ }^{1}$ \\ 'Instituto de Óptica "Daza de Valdés", Consejo Superior de Investigaciones Científicas, \\ C/Serrano 121, 28006 Madrid, Spain \\ ${ }^{2}$ Laboratorio de Ensayos. Centro de Investigación y Desarrollo de la Armada (CIDA), \\ C/Arturo Soria 289, 28033 Madrid, Spain \\ ${ }^{3}$ Institute of Physics, Wroclaw University of Technology, \\ Wybrzeze Wyspianskiego 27, 50370 Wroclaw, Poland \\ *Corresponding author: sortiz@io.cfmac.csic.es
}

Received 22 October 2009; accepted 5 November 2009;

posted 13 November 2009 (Doc. ID 118609); published 2 December 2009

\begin{abstract}
We address the measurement and correction of fan distortion in optical coherence tomography (OCT). This effect arises from the scanning system configuration and prevents one, in general, from obtaining quantitative topographic data from OCT. Computer simulations allowed us to quantify the effect and evaluate its dependence on the scanning mirror separation and design of the collimating lens, as well as to estimate the optimal axial position of that lens to minimize the fan distortion. We also developed a numerical algorithm based on 3-D ray propagation for the correction of the residual fan distortion. The effect was studied experimentally using a custom developed time-domain OCT in a Michelson configuration provided with a confocal channel, and the accuracy of the fan distortion correction algorithm tested on samples of known dimensions (flat surfaces and spherical lenses). With a proper calibration of the system with use of an onfocal channel, this algorithm makes it possible for time-domain OCT devices to be used as topographers. (C) 2009 Optical Society of America
\end{abstract}

OCIS codes: $\quad 110.4500,120.6650,120.4640,110.6880,330.7327,330.4300$.

\section{Introduction}

Optical coherence tomography (OCT) is a noninvasive imaging technique developed in 1991 [1,2]. Essentially it consists of an interferometer that uses a low coherence light source to gate the space $[3,4]$. This way all the samples that fall out of the path matching the path in the reference arm of the interferometer do not produce interference fringes. Therefore, only signals returning from a particular location in the sample are captured by the device. Resolution (i.e., the ability to resolve two consecutive sample layers) [5] is inversely proportional to the light source ban $\bar{d}$ width [6]. Examples of light sources used in OCT de-

0003-6935/09/356708-08\$15.00/0

(C) 2009 Optical Society of America vices include superluminescent diodes (SLDs) with maximum resolution of $10-15 \mu \mathrm{m}$, femtosecond laser with resolutions of $2-3 \mu \mathrm{m}$ [7], and swept sources that are able to provide resolution of $2-9 \mu \mathrm{m}$ [8].

The axial measurements in OCT are commonly named A-scans. Additionally, when scanners are added it is also possible to get images by rastering surfaces. The name of the imaging technique depends on the way of sweeping the surface. If it is a collection of A-scans that, when put together, form a cross section of the sample, it is denoted a B-scan. However, if the sample is rastered first and after the reference arm is moved, the technique is named a C-scan or en-face [9]. Three-dimensional images can be obtained straightforwardly using any of the rastering techniques introduced before [10]. Its inherent ability to resolve differences in distance in 
the micrometer domain and the possibility of introducing fast optical scanners make OCT a perfect tool to act as a topographer. So far there are only a few examples in the literature using OCT for quantitative surface characterization $[11,12]$. The main limitation for the direct quantitative analysis of surfaces is so-called fan distortion. Fan distortion is a set of aberrations associated with the scanning system architecture of the optical setup that typically consists of two-mirror and two-axis scanning systems [1316]. Such scanning systems are used in most OCT devices. Field distortion and astigmatism make the greatest contributions to the magnitude of fan distortion. These Seidel aberrations result in deformation of the image of the tested sample [17,18], and in OCT devices they arise primarily from the separation of the mirrors in the mentioned two-axis scanning system (see Fig. 1). The other contributions to the distortion arise from the precision of the flatness of the scanner mirrors [17] or slight misalignment of the light beam with the axis of rotation of the mirrors. However, with a proper calibration of the fan distortion introduced by the optical scanner, OCT can be used as a tool to retrieve the geometrical parameters of surfaces. Some authors [19] have addressed fan distortion in an OCT system, in which the rastering was produced by a nonlinear scanning system (consisting of resonant mirrors plus

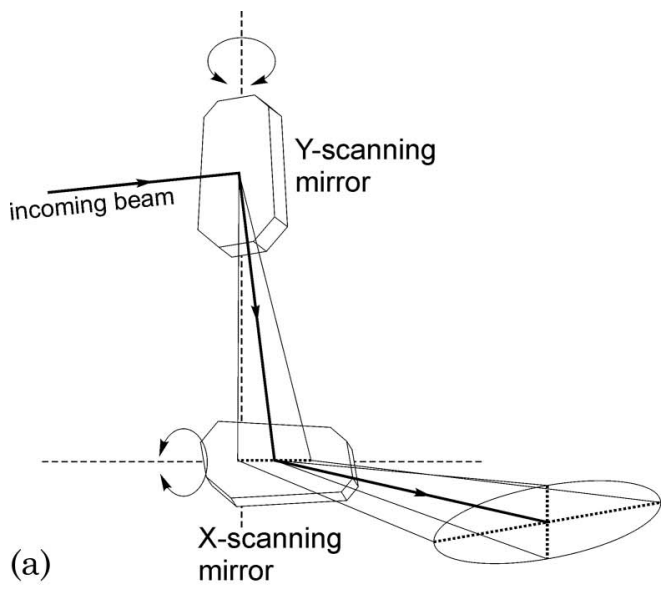

(a)

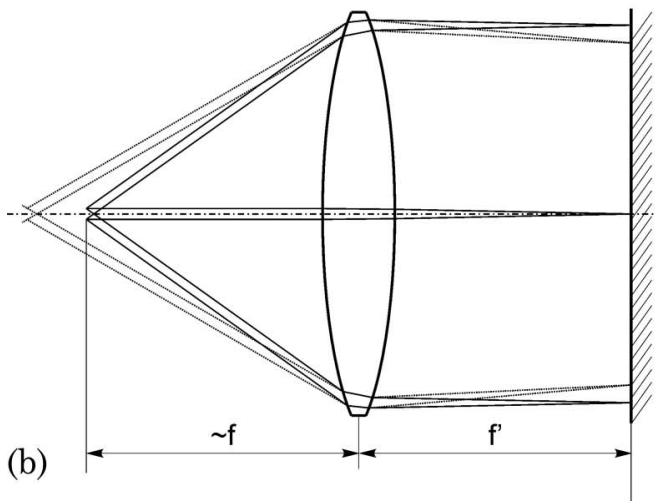

Fig. 1. Separation of mirrors in the scanner (a) results in a different beam deflections after being refracted by a collimating lens (b) in an OCT setup. nontelecentric scanning). They proposed to correct the distortion with the use of images taken axially around the confocal position of the beam, without taking into account the 3-D information provided by the OCT. Therefore the proposed procedure can be applied only to 2-D imaging, not to 3-D topography. Therefore the topic still seems to be open and a solution timely, as current OCT systems start allowing real-time 3-D images acquisition in living samples.

In the current study we will present a custom timedomain OCT system developed for in vitro anterior chamber measurements with an additional real-time OCT channel that enables reliable calibration of the system and relatively fast alignment of the tested sample (preview feature). A proper and reliable calibration is critical if the OCT setup is to be used for quantitative measurements of the surface topography. We also present some experimental results of topographic measurements of test samples of known geometry, in particular intraocular lenses (IOLs).

\section{Methods}

\section{A. Modeling of the Fan Distortion}

When imaging even perfectly flat surfaces by means of 3-D OCT devices, they become curved. This phenomenon can be described as a combination of at least two possible effects: (1) architecture of the scanning system, with relatively significant influence of spatial separation of the mirrors and (2) design, position, and alignment of the collimation lens in relation to the mirrors of the scanner.

There exist a variety of designs and architectures of 2-D scanning systems [20]. One feature that is common to most of them is that the mirrors responsible for angular beam deflection are separated by some distance. In a preobjective scanning configuration, this is equivalent to the situation presented in Fig. 1(b), where the origins of the fan in sagittal and meridional cross sections (solid and dotted lines, respectively) are separated by the distance between mirrors in the scanner. Therefore, the incident angles of the marginal beams in the two perpendicular cross sections are different. This configuration results in different exit angles of off-axis beams (the further from the optical axis, the larger the difference in angle). This leads to relevant differences in the optical path, which finally result in the distortion of a perfectly flat surface that can be seen in raw OCT images. The effect is shown schematically in Fig. 2, which presents two OCT images of a flat optical surface (mirror) captured in two perpendicular directions. In this figure the effect of fan distortion can be clearly observed: the flat surface becomes curved, and in one cross section the curvature is larger than in the other.

The design, position, and alignment of the collimating lens have a very significant impact on the magnitude of fan distortion. We developed an algorithm in MATLAB for simulations of the beam 

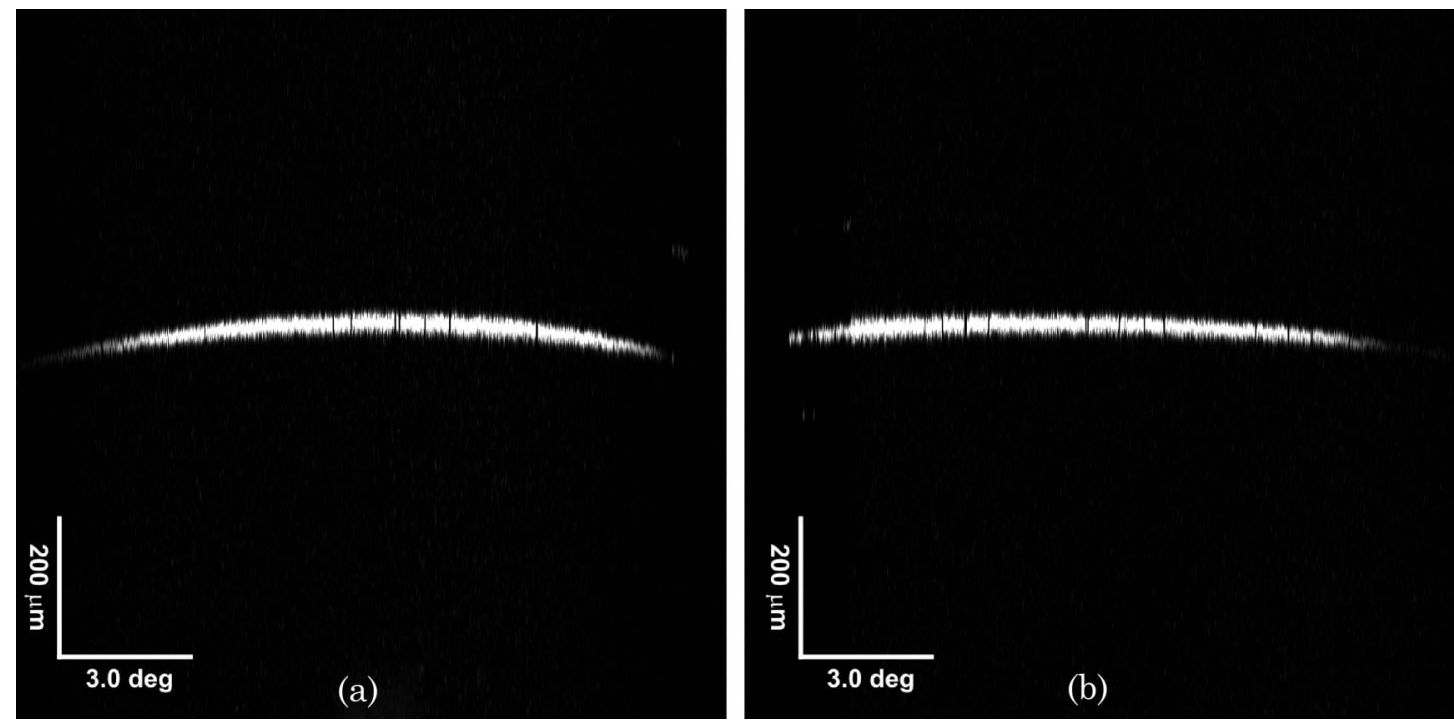

Fig. 2. OCT images of a flat optical surface (mirror) captured in two perpendicular directions: (a) vertical and (b) horizontal.

propagation in the OCT sample arm of the interferometer toward the sample. In this algorithm it was assumed that small-diameter beams used in the OCT scanning systems can be well described by single rays and that the rays are propagated according to the laws of geometric optics. In order to perform realistic simulations, the algorithm can take into account the eventual noncoaxial alignment of the scanner mirrors with respect to the chief ray, various separations between the scanning mirrors, and various locations of the collimating lens (regarded as a thick lens) that focuses the beam on the sample. The algorithm is able to estimate the optimal lens position by minimizing the rms of the average value of the directional cosines of propagation vectors behind the lens. Experimentally, this can be achieved by moving the lens along the optical axis. It should be noticed that, if the system is free of aberrations, the propagation vectors of the rays are perfectly parallel to each other and their rms is equal to zero.

\section{B. Experimental Setup}

In order to evaluate the fan distortion experimentally, a classical time-domain Doppler shifted OCT setup was developed. The system was built in a free air Michelson interferometer configuration. The low coherence light source was a SLD (Superlum, Carrigtwohill, Ireland) with a central wavelength of $820 \mathrm{~nm}$ and a $20 \mathrm{~nm}$ bandwidth. The light coming from the SLD was collimated by a pigtailed lens (Princetel, Pennington, New Jersey, USA). The reference arm consists of a stepper motor (Thorlabs, Dachau, Germany) configured with a speed of $23.75 \mathrm{~mm} / \mathrm{s}$, which provides a central Doppler frequency of $58.6 \mathrm{kHz}$. In the sample arm, a galvanometer scanner (Cambridge Technologies, Cambridge, Massachusetts, USA) of $3 \mathrm{~mm}$ clear aperture and a sampling frequency of $1 \mathrm{kHz}$ was used together with plano-convex lens (Newport, Irvine, California, USA) of $75 \mathrm{~mm}$ focal length and $25 \mathrm{~mm}$ aperture as a collimation lens for chief rays. Finally, the light returned by the sample and reference arm is collected by a fiber optic pigtail, which drives the light to a balanced avalanche photodiode detector (Hamamatsu, Hamamatsu, Japan). The OCT signal is then filtered by a custom bandpass filter of central frequency of $58.6 \mathrm{kHz}$ and bandwidth of 2.4 and digitized by a PCI card (National Instruments, Austin, Texas, USA) and stored in the computer to produce the images.

A very useful feature of this setup is its capability to capture confocal images of the sample without any additional element. Taking advantage of the speed of the galvanometric scanner $(1 \mathrm{kHz})$ it is possible to use this feature for almost a real-time preview (about 2 frames per second with satisfying resolution of $400 \times 400$ pixels) of the sample. This confocal mode is very useful to align the sample correctly or to place it in the proper (focal) distance from the lens in order to optimize OCT signal-to-noise ratio. Moreover, with use of a confocal channel it is possible to calibrate the system and to estimate the magnitude of the distortion when a flat surface with a regular grid is imaged. The effects of 3-D and 2-D imaging of a flat surface will be illustrated in Section 3 .

\section{Estimation of the Residual Fan Distortion}

Even for the optimal lens position, there is some residual distortion that needs to be estimated and corrected numerically. For this purpose, a flat sample plate with a diffuse surface was used for calibration and tested on the experimental setup described in Subsection 2.B. This calibration plate contains a black grid on a white background with an interline separation of $1 \mathrm{~mm}$. Series of grid images through the exact confocal position of the sample were obtained using both the confocal and the 3-D OCT channels The images captured using the confocal channel were processed using a Hough transform [21] (a more efficient algorithm than simple edge 


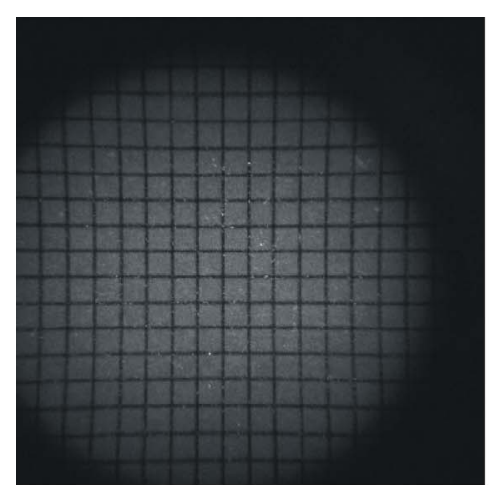

(a)

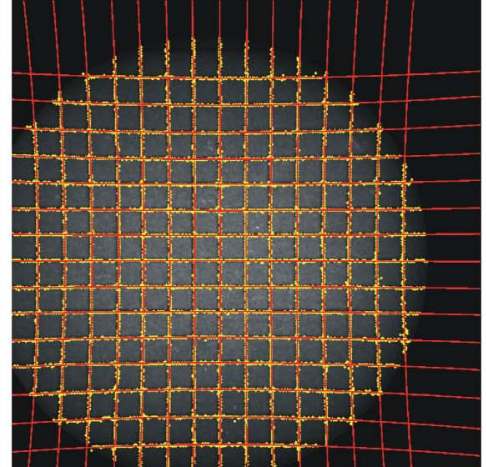

(b)

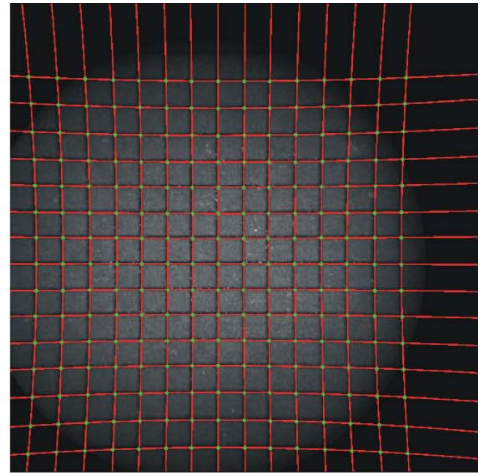

(c)

Fig. 3. (Color online) Steps of the calibration procedure using the confocal channel: (a) original confocal image, (b) grid line detection (points) and their approximation by parabolas (lines), (c) detection of the grid nodes (intersection points of the parabolas).

detection) to detect the lines of the grid in the image. The detected lines were fitted to parabolas (in the angular system of coordinates), and the intersections of vertical and horizontal parabolas were recognized as the nodes of the grid (the steps of processing the confocal data are presented in Fig. 3). This way each node can be expressed both in Cartesian coordinates $N_{i j}(x, y)$ and in angular coordinates $N_{i j}(\theta, \varphi)$, where $i$ and $j$ denote the number of row and column with respect to the reference node $(0,0)$, which is not affected by distortion. It should be noted that in the confocal image the angular system of coordinates is rectangular, as shown in Fig. 4. The conversion between these two systems is related to the length of the arc calculated for the equations of the parabolas given by $f(\theta)=a \theta^{2}+b \theta+c$ and $f(\varphi)=d \varphi^{2}+e \varphi+g$. These parabolas are least-mean-square approximations of the points detected on the confocal image and belonging to each horizontal and vertical grid line separately. Estimated node positions are computed as cross points of each horizontal parabola with each vertical one, which may be expressed by the following system of equations:

$$
N_{i j}(x, y)=\left\{\begin{array}{c}
x_{i}=\int_{0}^{\theta_{i}} \sqrt{1+f^{\prime}(\theta)} \mathrm{d} \theta \\
y_{i}=\int_{0}^{\varphi_{j}} \sqrt{1+f^{\prime}(\varphi)} \mathrm{d} \varphi
\end{array},\right.
$$

where $\left(x_{i}, y_{j}\right)$ and $\left(\theta_{i}, \varphi_{j}\right)$ are the coordinates of each node $N_{i j}$ in Cartesian and angular coordinates, respectively. This way, each point in the confocal image can be converted to the Cartesian coordinates by calculating

$$
P_{k l}(x, y)=\left\{\begin{array}{c}
x_{k}=\frac{\theta_{k}}{\Theta_{m}} X_{m} \\
y_{l}=\frac{\varphi_{l}}{\Phi_{n}} Y_{n}
\end{array},\right.
$$

where $\left(x_{k}, y_{l}\right)$ and $\left(\theta_{k}, \varphi_{l}\right)$ are the coordinates of the each point $P_{k l}$ in Cartesian and angular coordinates, respectively; $\left(X_{m}, Y_{n}\right)$ and $\left(\theta_{m}, \Phi_{n}\right)$ are the coordinates [related to reference point $(0,0)$ ] of the node $N_{m n}$ (in Cartesian and angular coordinates, respectively) that are closest to the point of interest $P_{i j}$. This conversion was used to obtain the angular input of the scanner into spatial coordinates.
The 3-D OCT images can be expressed in scanner angular coordinates plus a length to a reference point: $(\theta, \varphi, L)$, where $\theta$ is the elevation angle, $\varphi$ is the azimuth angle, and $L$ is the Euclidean distance (length) with respect to a reference point, being the minimum of the axial scanning range (to which any other axial scan range must be referred). The correspondence between the angular scanner coordinates and the spatial coordinates on the image is achieved through the conversion factor obtained for each axial location from confocal channel, as explained before, allowing us to express each OCT image of the series as $(x, y, L)$, where $x$ and $y$ are the horizontal and vertical coordinates, respectively, and $L$ is the uncorrected axial component (length). Correction of $L$ is achieved by $3-\mathrm{D}$ ray tracing in space. Rays can be described by the general ray equation in vectorial form:

$$
\vec{R}_{i}=O_{i}+L_{i} \hat{T}_{i}
$$

where $O_{i}$ is the origin point of the $i$ th ray, $L_{i}$ is the uncorrected axial component (length) for this ray, and $\hat{T}_{i}$ is the normalized directional cosine of this ray.

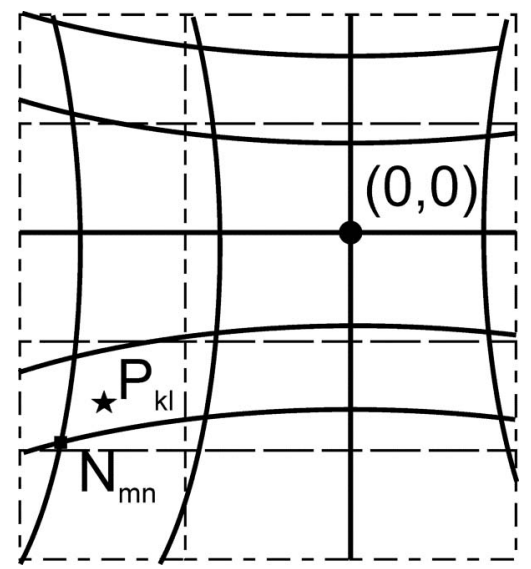

Fig. 4. Point $P_{k l}$ represented in angular (dashed lines) and Cartesian (solid lines) systems of coordinates. The origin of both systems is at the same point $(0,0)$ - the point not affected by distortion. Point $N_{m n}$ is the closest node to point $P_{k l}$. 
The directional cosines can be achieved by capturing a series of confocal images together with 3-D OCT measurements at each axial sample location around the confocal plane (the distances to the reference plane need to be known) and then identifying the coordinate positions of each pair of angles introduced into the scanner in each of the confocal images, tracking their relative movement spatial system coordinates (from one confocal image to the next one) as shown in Fig. 5(a). By fitting the $x_{i}$ and $y_{i}$ components to a line by a linear regression, the vertical and horizontal coordinates at the origin, as well as the components of the directional cosine can be obtained. By using regression analysis, the effect of the noise due to the introduction of the diffuse plate and to mechanical instability of the motor is minimized:

$$
\left\{\begin{array}{l}
x_{i}=x_{0}^{i}+L_{i} T_{x}^{i} \\
y_{i}=y_{0}^{i}+L_{i} T_{y}^{i}
\end{array}\right.
$$

where $x_{0}^{i}$ and $y_{0}^{i}$ are the horizontal and vertical coordinates of the origin for the $i$ th ray, respectively. Once the horizontal and vertical components of the directional cosine have been obtained, it is straightforward to get the axial component, since this vector is normalized. Finally, by selecting the origin of the axial component as 0 , the real axial coordinate can be obtained:

$$
z_{i}=L_{i} \sqrt{1-\left(T_{x}^{i}\right)^{2}+\left(T_{y}^{i}\right)^{2}} .
$$

\section{Fan Distortion Correction}

When the fan distortion of the setup is characterized, it can be easily removed using a general 3-D ray equation: a reference point in space $O$ and the direc-

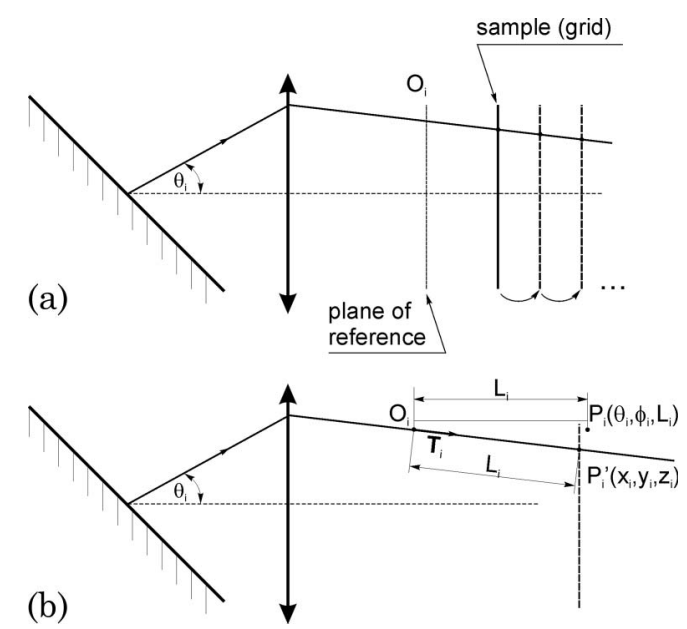

Fig. 5. (a) Capture of a series of confocal images at different axial grid positions for the purposes of system calibration. (b) Schematic explanation of the correction procedure. Point $P_{i}$ is obtained on the OCT and expressed in angular system of coordinates $(\theta, \varphi, L)$, where $\theta$ and $\varphi$ are the angles of the scanner, and $L$ is the distance from the reference point $O$ along the ray given by the propagation vector $T$. Point $P_{i}^{\prime}$ is the point after correction in Cartesian coordinates. tional cosine of a ray at this point $\hat{T}$. Any point at this ray can be placed by simple substitution of the angles in Eq. (4). If the particular scanner angle was not measured experimentally, the correspondence can be obtained from a bicubic interpolation of the measured angle. Therefore, since the scanning beam can be approximated by a single ray, and each pair of angles of the scanning mirrors is related exactly to one normalized vector of propagation $\hat{T}_{i}$, the information achieved from the calibration of the setup (from the scheme presented above) can be used to obtain the coordinates of the normalized vector of propagation $\hat{T}_{i}$. Once it is obtained, the corrected coordinates of the point $P_{i}{ }^{\prime}$ on a surface can be found by multiplying the distance obtained from OCT $L_{i}$ by the directional cosine vector and adding the reference point for this specific ray:

$$
P_{i}^{\prime}=O_{i}+L_{i} \hat{T}_{i}
$$

Figure 5(b) presents the schematic explanation of the method.

A useful feature of this algorithm based on ray propagation expressed in angular notation is that the distortion is constant and does not depend on the axial position of the sample, unlike the Cartesian coordinates. For this purpose, the shape of the examined samples in the uncorrected OCT images does not change with the axial position of the sample (however, the image quality may change).

\section{E. Validation on Surfaces of Known Geometry}

In order to validate the fan distortion correction method, its accuracy was tested on several surfaces of known geometries. These surfaces were (1) a flat optical surface, (2) a spherical lens (used in previous studies as an artificial cornea [22]), and (3) a biconvex commercial poly methyl(methacrylate) (PMMA) spherical IOL (anterior and posterior surface).

The spherical surfaces were measured additionally by confocal microscopy based noncontact profilometry (PL $\mu$, Sensofar-Tech SL, Terrasa, Spain) in order to have the values of reference. Each surface was measured using the described OCT setup (55 Bscans, 55 A-scans each). The angular dimensions used to scan the surface differed across samples: -4.5 to $4.5^{\circ}$ for the flat surface, -3.3 to $3.3^{\circ}$ for the artificial cornea, and -1.5 to $1.5^{\circ}$ for the IOLs. The 3-D data were processed with use of custom algorithms for line detection and surface identification. Once the cloud of points belonging to every surface was identified, the algorithm to correct fan distortion was applied and then the points were fitted by analytical equations of spheres using a least-meansquare algorithm. The fits were performed on the raw points, as well as on the set points after fan distortion correction. The radii of curvature of each surface were obtained and compared to the values with the use of a noncontact profilometer. 
(a)

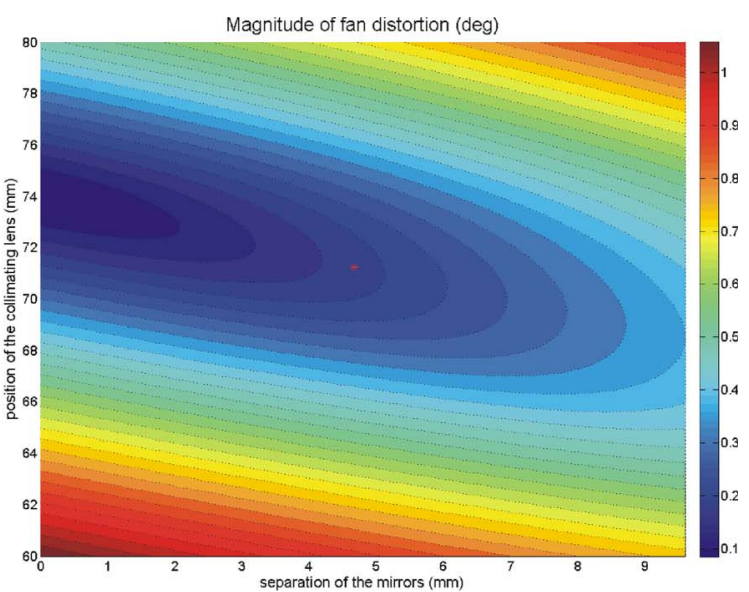

(b)

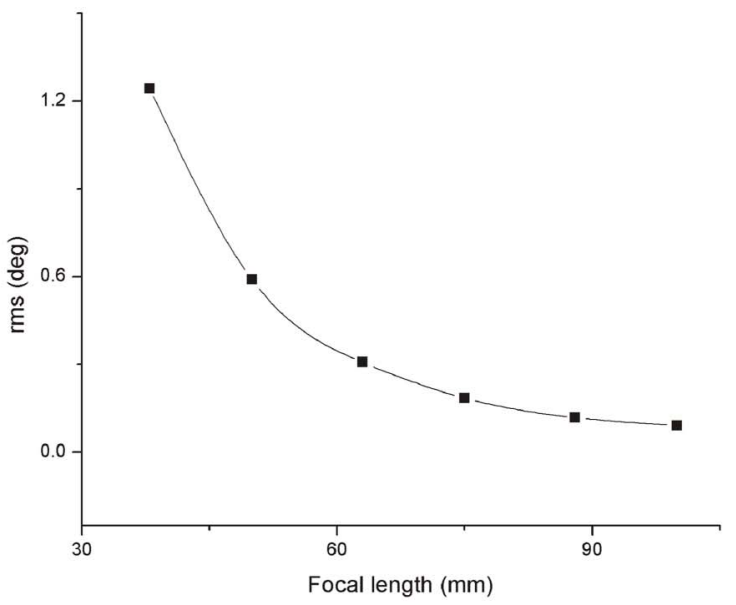

(c)

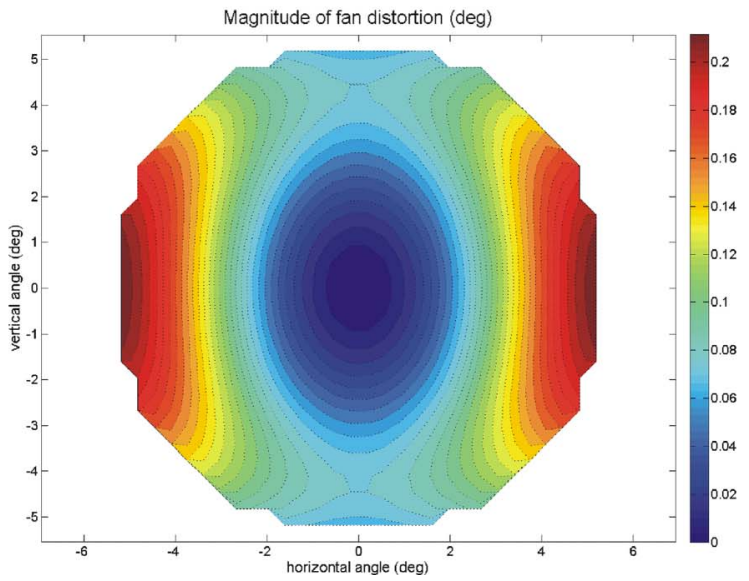

Fig. 6. (Color online) Results of simulations of the fan distortion: (a) distortion as a function of the lens position and separation between scanner mirrors, (b) distortion as a function of the focal length of the collimating lens (in its optimal position), and (c) fan distortion for the architecture used in the experiment.

\section{Results}

\section{A. Simulation of Fan Distortion}

We introduced the parameters of our OCT system in the simulation presented in Subsection 2.A and applied a minimization algorithm to determine the optimal axial location of the collimating lens that provided the lowest level of fan distortion. The effect

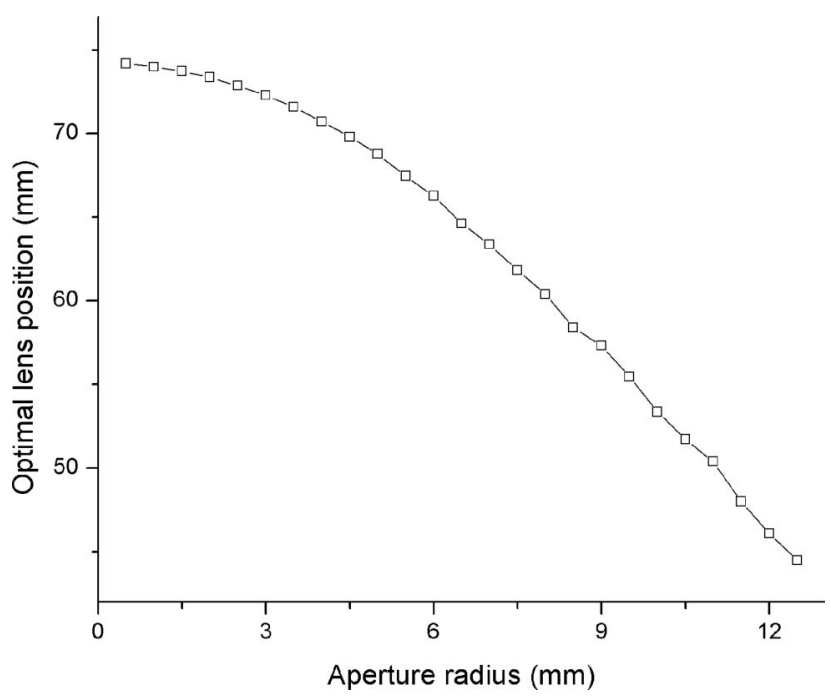

Fig. 7. Optimal lens position as a function of lens aperture radius.

of the separation between the scanning mirrors and the focal length of the collimating lens was also tested.

The most relevant geometric parameters for the model are the distance between centers of mirrors and collimating lens $(4.67 \mathrm{~mm})$ and the radii of curvature $(39.070 \mathrm{~mm}$ and infinity), thickness $(5.122 \mathrm{~mm})$, and refractive index of BK7 (for $0.820 \mu \mathrm{m}$ wavelength) of the collimated lens. Simulations were performed for a random pattern of 200 rays in the 3-D ray tracing.

Figure 6(a) presents the results of simulation of the magnitude of aberrations (rms) as a function of the position of the lens and of the separation between the scanning mirrors. The magnitude of the distortion is expressed in terms of the rms of the ray deviations with respect to theoretical collimation. The position of the lens is referred to the position of the vertical scanning mirror. The global minimum is found for a telecentric configuration, which is possible only for scanning in one direction (B-scan). For any other distance between the scanning mirrors, there exists one position of the collimating lens that corresponds to the minimum of the rms for this particular configuration. This means that the optimal position depends strongly on the separation distance between the scanner mirrors and the design of the lens (which affects its spherical aberration). Figure 6(a) shows the magnitude of the fan distortion as a function of the focal length of the collimating lens placed at its optimal position. Figure $6(\mathrm{~b})$ presents a 3 -D plot of the fan distortion simulated for the architecture used in our experimental system. Points on this plot correspond to the beams having the same optical path. The astigmatic shape of this distortion arises from the separation of the scanner mirrors: a larger separation results in a larger peak-to-valley difference of the distortion. For the collimating lens of our OCT system and the scanning mirror separation in our galvanometer system the optimal position 
Table 1. Radii of Curvature of Tested Surfaces as Measured with Noncontact Profilometry and OCT (Before and After Distortion Correction)

\begin{tabular}{lccc}
\hline Type of Data & Profilometer & $\begin{array}{c}\text { OCT Before } \\
\text { Correction }^{a}\end{array}$ & $\begin{array}{c}\text { OCT After } \\
\text { Correction }\end{array}$ \\
\hline $\begin{array}{l}\text { Anterior IOL } \\
\text { surface (mm) }\end{array}$ & 17.66 & 17.40 & 17.70 \\
$\begin{array}{l}\text { Posterior IOL } \\
\text { surface (mm) }\end{array}$ & 10.70 & 10.86 & 10.76 \\
$\begin{array}{l}\text { Artificial cornea } \\
(\mathrm{mm})\end{array}$ & 7.76 & 8.73 & 7.77 \\
\hline
\end{tabular}

${ }^{a}$ Values calculated by simple conversion from angular to Cartesian coordinates.

for the collimated lens [marked with an asterisk in Fig. 6(a)] was $71.42 \mathrm{~mm}$ from the vertical scanner mirror.

We found that the optimal lens position was also dependent on the aperture size, due to the influence of the spherical aberration of the lens on the fan distortion. We repeated the simulation for different apertures (radius ranging from of $1 \mathrm{~mm}$ to full aperture of the lens in $1 \mathrm{~mm}$ steps). Figure 7 shows the dependence of the optimal distance on lens aperture, as predicted from the simulations.

\section{B. Residual Fan Distortion in the Experimental System}

In the experimental setup we placed the lens at the optimal distance estimated from the simulations, as both the lens parameters and scanner architecture of the experimental system were used in the model. Despite obvious technical difficulties in setting the exact distance, the reconstructed OCT data of a flat surface (optical mirror) are very similar to the simulated data [presented in Fig. 6(c).], confirming the predictions from the model.

\section{Correction of Fan Distortion of OCT Images}

The developed fan correction distortion algorithm was applied to the correction of residual distortion

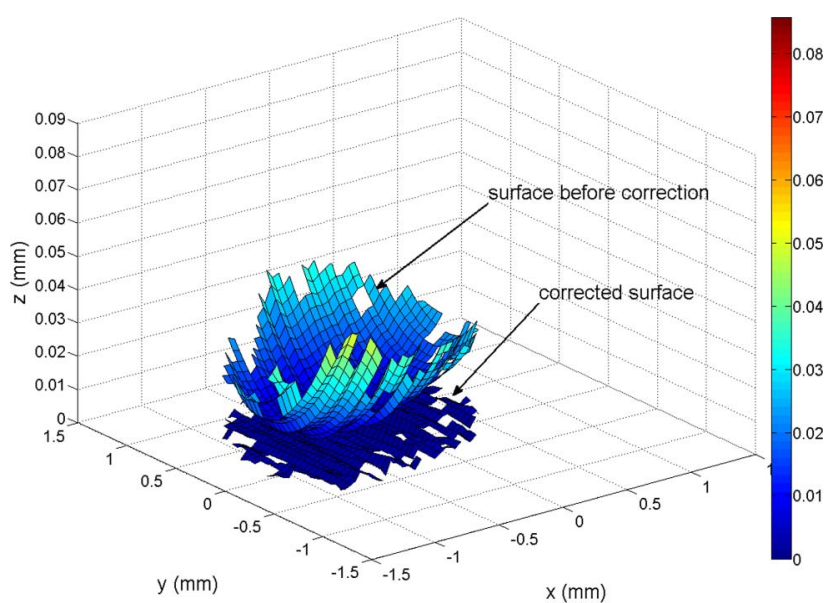

Fig. 8. (Color online) Comparison of topographies of a flat surface obtained by means of OCT before and after numerical correction. The shape of the uncorrected surface is given by simple conversion from angular to Cartesian coordinates. in 3-D OCT images of a flat surface, shown in Fig. 2. Figure 8 shows the efficiency of the correction applied to the distortion data of a flat surface. The deviation from flatness of the corrected surface is very small ( $\mathrm{rms}<2 \mu \mathrm{m}$, while the peak-to-valley magnitude of the distortion was about $90 \mu \mathrm{m}$ ). The correction of the residual fan distortion was also applied to three PMMA spherical surfaces of known radii of curvature. The results are shown in Table 1 . Raw elevation data, profilometric data, and elevation data after fan distortion correction were fitted by spherical surfaces using the same fitting algorithm.

\section{Conclusions}

In the present study we have described the phenomenon of fan (field) distortion, which is associated with the architecture of the scanning system and alignment of its optical elements. We have shown that the presence of a fan causes distortions in an OCT system and proposed a method for correction. The effect has been shown on a custom laboratory time-domain OCT system in which a confocal channel has proved a very useful tool for calibration and sample alignment.

The magnitude of fan distortion depends on the distance between scanning mirrors, as well as the position of the collimation lens and its power and aperture. Fan distortion was minimized by positioning the collimated lens according to predictions from the 3-D ray tracing model. Fan distortion can be also reduced by increasing the focal length of the collimating lens. Algorithms to correct the residual fan distortion have also been proposed and applied to real surfaces imaged with the OCT device. After correction, we showed discrepancies of less than $2 \mu \mathrm{m}$ rms flatness on flat surfaces and less than $0.2 \%$ in the radii of curvature of spherical lenses with respect to values measured with another method, while the uncorrected data showed discrepancies up to $90 \mu \mathrm{m}$ for flat surface and up to $12 \%$ in the radii of curvature. We conclude that after application for fan distortion OCT can be reliably used for topography of optical surfaces. OCT systems may be more versatile than other noncontact profilometry devices, as the former can work on both transparent and diffusing surfaces, and the lateral range of application is limited only by the angular range of the galvanometric scanners. The lateral resolution is hardware limited and depends mainly on the sample beam diameter and optics and the quality of scanners used in the setup, while the axial resolution depends on the light source bandwidth and subpixel precision of the edge detection algorithms. Also, with some modifications in the calibration method (based on other approaches rather than the use of a confocal channel), the fan distortion correction algorithms described here could also be applied to spectral OCT systems, which could then be used as reliable corneal topographers [23,24].

As fan distortion arises from the aberrations associated with the architecture of the scanning system, it is also influenced by misalignment of the optical elements of the system. While we have tested 
(theoretically and experimentally) the case of a wellcentered instrument, the proposed methods can also evaluate the possible effect of deviations of the beam from the pivot axis of the scanning mirrors.

Although most OCT systems use 2-axis galvanometric scanning systems to raster the sample (and this is the configuration that we have simulated and tested experimentally), an alternative to minimize fan distortion can be the use of scanning systems that do not show a separation between mirrors (i.e., microelectromechanical systems technology $[25,26])$. In this case, the magnitude of fan distortion will depend only on the spherical aberration of the focusing/collimating lens and its alignment, and our proposed model and algorithm will still be applicable to predict and compensate distortion. To our best knowledge all commercial OCT devices used for anterior chamber imaging use 2-axis galvanometric systems, and the fan distortion is expected to be a significant limiting factor for quantitative measurements of corneal topography. The methods presented in this study can be generalized to be applied to improve the reliability of OCT systems as corneal topographers.

This research was funded by grants FIS200802065 and PETRI PET-2006-0478 (Ministerio de Ciencia e Innovación, Spain) to Susana Marcos; EURYI-05-102-ES (EURHORCs-ESF) to Susana Marcos; CSIC JAE-Programs to Damian Siedlecki and Laura Remón; and Alcon, Spain, to Sergio Ortiz. The authors thank Daniel Pascual for technical assistance in building the OCT system and Noelia Chía for technical assistance in data collection and processing.

\section{References}

1. D. Huang, J. Wang, C. P. Lin, C. A. Puliafito, and J. G. Fujimoto, "Micron-resolution ranging of cornea anterior chamber by optical reflectometry," Lasers Surg. Med. 11, 419-425 (1991).

2. D. Huang, E. Swanson, C. P. Lin, J. S. Schuman, W. G. Stinson, W. Chang, M. R. Hee, T. Flotte, K. Gregory, C. A. Puliafito, and J. G. Fujimoto, "Optical coherence tomography," Science 254, 1178-1181 (1991).

3. A. F. Fercher, W. Drexler, C. K. Hitzenberger, and T. Lasser, "Optical coherence tomography-principles and applications," Rep. Prog. Phys. 66, 239-303 (2003).

4. J. M. Schmitt, "Optical coherence tomography (OCT): a review," IEEE J. Sel. Top. Quantum Electron. 5, 1205-1215 (1999).

5. B. Grajciar, M. Pircher, C. K. Hitzenberger, O. Findl, and A. F. Fercher, "High sensitive measurement of the human axial eye length in vivo with Fourier domain low coherence interferometry," Opt. Express 16, 2405-2414 (2008).

6. M. Brzezinski, Optical Coherence Tomography: Principles and Applications (Academic, 2006).

7. U. Morgner, F. X. Kärtner, S. H. Cho, Y. Chen, H. A. Haus, J. G. Fujimoto, E. P. Ippen, V. Scheuer, G. Angelow, and T. Tschudi, "Sub-two-cycle pulses from a Kerr-lens mode-locked Ti:sapphire laser," Opt. Lett. 24, 411-413 (1999).

8. C. Lu, M. Tsai, Y. Wang, C. Lee, Y. Kiang, and C. C. Yang, "High-resolution swept-source optical coherence tomography with the frequency-sweeping the broadened spectrum of a fs Cr:forsterite laser," in Conference on Lasers and ElectroOptics/Quantum Electronics and Laser Science Conference and Photonic Applications Systems Technologies (Optical Society of America, 2007), paper JTuA44.

9. A. G. Podoleanu, G. M. Dobre, and D. A. Jackson, "En-face coherence imaging using galvanometer scanner modulation," Opt. Lett. 23, 147-149 (1998).

10. R. B. Rosen, M. Hathaway, J. Rogers, J. Pedro, P. Garcia, P. Laissue, G. M. Dobre, and A. G. Podoleanu, "Multidimensional en-face OCT imaging of the retina," Opt. Express 17, 4112-4133 (2009).

11. E. Kim, K. Ehrmann, S. Uhlhorn, D. Borja, and J.-M. Parel, "Automated analysis of OCT images of the crystalline lens," Proc. SPIE 7163, 716313 (2009).

12. A. Podoleanu, I. Charalambous, L. Plesea, A. Dogariu, and R. Rosen, "Correction of distortions in optical coherence tomography imaging of the eye," Phys. Med. Biol. 49, 1277-1294 (2004).

13. Y. Li, "Laser beam scanning by rotary mirrors. II. Conicsection scan patterns," Appl. Opt. 34, 6417-6430 (1995).

14. Y. Li and J. Katz, "Asymmetric distribution of the scanned field of a rotating reflective polygon," Appl. Opt. 36, 342-352 (1997).

15. G. F. G. Marshall, "Scanning devices and systems," in Applied Optics and Optical Engineering, R. Kingslake and B. J. Thompson, eds. (Academic, 1980), Vol. 6, pp. 203-262.

16. Y. Li, "Beam deflection and scanning by two-mirror and twoaxis systems of different architectures: a unified approach," Appl. Opt. 47, 5976-5985 (2008).

17. J. Xie, S. Huang, Z. Duan, Y. Shi, and S. Wen, "Correction of the image distortion for laser galvanometric scanning system," Opt. Laser Technol. 37, 305-311 (2005).

18. M. F. Chen and Y. P. Chen, "Compensating technique of field-distorting error for the $\mathrm{CO}_{2}$ laser galvanometric scanning drilling machines," Int. J. Mach. Tools Manuf. 47, 1114-1124 (2007).

19. V. Westphall, A. M. Rollins, S. Radhakrishnan, and J. A. Izatt, "Correction of geometric and refractive image distortions in optical coherence tomography applying Fermat's principle," Opt. Express 10, 397-404 (2002).

20. J. I. Montagu, "Galvanometric and resonant scanners," in Handbook of Optical and Laser Scanning, G. F. Marshall, ed. (Marcel Dekker, 2004), pp. 417-476.

21. L. A. F. Fernandes and M. M. Oliveira, "Real-time line detection through an improved Hough transform voting scheme," Pattern Recogn. 41, 299-314 (2008).

22. A. de Castro, P. Rosales, and S. Marcos, "Tilt and decentration of intraocular leses in vivo from Purkinje and Scheimpflug imaging-a validation study," J. Cataract Refract. Surg. 33, 418-429 (2007).

23. M. Gora, K. Karnowski, M. Szkulmowski, B. J. Kaluzny, R. Huber, A. Kowalczyk, and M. Wojtkowski, "Ultra high-speed swept source OCT imaging of the anterior segment of human eye at $200 \mathrm{kHz}$ with adjustable imaging range," Opt. Express 17, 14880-14894 (2009).

24. Grulkowski, M. Gora, M. Szkulmowski, I. Gorczynska, D. Szlag, S. Marcos, A. Kowalczyk, and M. Wojtkowski, "Anterior segment imaging with spectral OCT system using a high-speed CMOS camera," Opt. Express 17, 4842-4858 (2009).

25. T. Zhou, P. Wright, J. Crawford, G. Mckinnon, and Y. Zhang, "MEMS 3-D optical mirror/scanner," in Proceedings of the 2003 International Conference on MEMS, NANO and Smart Systems (IEEE Computer Society, 2003), pp. 222-226.

26. Y. Xu, J. Singh, C. S. Premachandran, A. Khairyanto, K. W. S. Chen, N. Chen, C. J. R. Sheppard, and M. Olivo, "Design and development of a 3-D scanning MEMS OCT probe using a novel SiOB package assembly," J. Micromech. Microeng. 18, 125005 (2008). 